\title{
Impact of Surgically and Radiologically Detected Incidental Internal Mammary Lymph Node Enlargement in Breast Cancer Patients Undergoing Free-Flap Breast Reconstruction
}

Haitham H. Khalil, MSc, MRCS, MD, FRCS ${ }^{1}$ Maninder Kalkat, MS, MCh, FRCS² Marco N. Malahias, MSc, MRCS ${ }^{1}$ Saif Rhobaye, MRCS, MD ${ }^{1}$ Tarek Ashour, MSc, MRCS, MD, EBOPRAS ${ }^{1,3}$ Tahir Faroq, FRCS ${ }^{4}$ Amar Shimal, MRCP, FRCR ${ }^{5}$ Medy Tsalic, MD ${ }^{6}$ Babu Naidu, MSc, MD, FRCS ${ }^{2}$

${ }^{1}$ Department of Oncoplasty and Reconstructive Surgery, Good Hope Hospital, University Hospitals of Birmingham, Birmingham, West Midlands, United Kingdom

2 Department of Thoracic Surgery, Heartlands Hospital, University Hospitals of Birmingham, Birmingham, West Midlands, United Kingdom

3 Department of Plastic and Reconstructive Surgery, Cairo University Hospitals, Cairo, Egypt

${ }^{4}$ Department of Breast Surgery, Good Hope Hospital, University Hospitals of Birmingham, Birmingham, West Midlands, United Kingdom

${ }^{5}$ Department of Radiology, Good Hope Hospital, University Hospitals of Birmingham, Birmingham, West Midlands, United Kingdom

${ }^{6}$ Department of Oncology, Solihull Hospital, University Hospitals of Birmingham, Solihull, West Midlands, United Kingdom
Address for correspondence Haitham H. Khalil, MSc, MRCS, MD, FRCS, Department of Oncoplasty and Reconstructive Surgery, Good Hope Hospital, University Hospitals of Birmingham, Rectory Road, Sutton Coldfield, Birmingham B75 7RR, West Midlands, United Kingdom (e-mail: haitham.khalil@heartofengland.nhs.uk).

J Reconstr Microsurg Open 2018;3:e32-e40.

\begin{abstract}
Keywords

- internal mammary lymph node

- breast cancer

- breast reconstruction

- free flap
\end{abstract}

Background The internal mammary lymph node (IMLN) basin is considered the second most important regional nodal basin in breast cancer. IMLNs are often not detected radiologically and left untreated, with symptomatic recurrence being $0.1 \%$. Challenges in accessibility have been an obstacle in achieving a comprehensive treatment plan, especially with undetermined and radiologically enlarged IMLN. Free autologous tissue breast reconstruction is considered the gold standard, and the familiarity of microvascular surgeons in using the internal mammary vessels (IMVs) puts them in a unique position to shed more light on the natural pathological process of IMLN metastases.

Materials and Methods A retrospective data analysis study was conducted evaluating 270 patients who underwent 307 free flaps for breast reconstruction using the IMV in the period between 2009 and 2017. Patient's demographics and clinicopathological data including IMLN harvest, radiological, operative details, adjuvant therapy, postoperative morbidity, and follow-up outcome data were analyzed.

Results Eighty-nine enlarged IMLNs were surgically retrieved from 30.7\% (83/270) of the patients ( 73 delayed, 10 immediate breast reconstructions) with an age range of 29 to 77 years (mean: 45). Eighty six were incidentally encountered during surgery, whereas in three, the enlarged IMLN was preoperatively, radiologically determined and biopsied during computed tomography (CT) scan staging and was retrieved received

September 30, 2017 accepted after revision March 5, 2018
DOI https://doi.org/

10.1055/s-0038-1642626. ISSN $2377-0813$
Copyright $\odot 2018$ by Thieme Medical Publishers, Inc., 333 Seventh Avenue, New York, NY 10001, USA. Tel: +1(212) 584-4662.
License terms

(c) (1) $\ominus$ (\$) 
subsequently during surgery. IMLN metastases were confirmed in $8.4 \%$ (7/83) of the patients in whom IMLNs were retrieved with subsequent modification of the proposed adjuvant therapy. The follow-up period ranged from 3 to 84 months (mean: 42 ) for the involved IMLN patients. Two patients (28\% [2/7]) showed signs of disease progression with mortality.

Conclusion Microvascular surgeons in a multidisciplinary setting would provide a valuable role in improving outcomes of patients with IMLN metastases through better diagnosis and staging of incidentally enlarged metastatic IMLN and provision of an effective approach for locoregional disease control.

The internal mammary lymph node (IMLN) basin is considered the second most important regional nodal basin region in breast cancer. ${ }^{1,2}$ Lymphatic mapping studies revealed that pathological IMLN involvement enlargement could be in 10 to $15 \%$ of breast cancer patients, with up to $20 \%$ of these being positive for malignancy. ${ }^{3,4}$ Respectively, there have been contrasted views that had led to dilemma and controversy regarding the significance of sampling these nodes and their impact on staging, survival, and subsequent adjuvant therapy. Most of the studies have been limited to descriptive observations of early experiences and have led to inconsistent results with insufficient evidence in terms of efficacy and clinical significance. ${ }^{4-12}$ However, meta-analysis by the Early Breast Cancer Trialists' Collaborative Group showed poor outcomes in patients with IMLN involvement, leading to a resurgence of interest in the IMLN mapping and biopsy. ${ }^{9,13}$ Involved IMLNs are often not detected and left untreated, with symptomatic recurrence in this chain seen in $0.1 \%$ of breast cancer patients. ${ }^{14}$ The challenges in accessibility in terms of IMLN biopsy or dissection have been an obstacle in the widespread adoption of this technique. The familiarity of microvascular surgeons in using the internal mammary vascular pedicle (IMVP) as recipient vessels for free autologous tissue transfer for breast reconstruction puts them in a unique position to shed more light on the pathological process of IMLN metastases and recurrence.

We report our case series of incidentally diagnosed metastatic IMLN in breast cancer patients undergoing free-flap breast reconstruction using internal mammary vascular pedicle (IMVP) and the impact on their oncological management.

\section{Materials and Methods}

A retrospective analysis review of patients who underwent free autologous tissue transfer for breast reconstruction (immediate breast reconstruction [IBR] or delayed breast reconstruction [DBR] setting) postmastectomy in the period between January 2009 and March 2017 at Good Hope and Heartlands Hospitals, Birmingham, United Kingdom, was performed. The patients were identified from the department's audit database, and subsequently all patients who had incidental enlarged IMLNs were identified from the operative details, and radiological and histopathology database. Patients were managed through a multidisciplinary team approach
(Breast Oncoplasty MDT) comprising an oncologist, a radiologist, breast surgeons, a reconstructive surgeon (H. K.), and a thoracic surgeon (M. K.). In addition, the demographic information and clinicopathological details, including timing of reconstruction, adjuvant therapy, postoperative morbidity, disease-free interval, survival, and follow-up period data, were collected from the electronic patient records and analyzed. As per the standard protocol for preoperative oncological staging, all immediate reconstruction patients underwent standalone sentinel lymph node biopsy (SLNB) of the axilla. On the other hand, patients requesting delayed reconstruction and who were deemed moderate or high risk for locoregional or distant metastases were candidates for staging computed tomography (CT) scan. If an incidental enlarged IMLN was detected radiologically, the patient was subjected to positron emission tomography (PET)/CT scan or mediastinoscope-guided biopsy prior to any definitive reconstruction ( - Fig. 1). The IMVP was the recipient vessel of choice in all patients undergoing microvascular free-flap breast reconstruction either in an immediate or delayed setting. The IMVP was approached through dissection of the third costal cartilage, and any macroscopically enlarged IMLN was harvested and subjected to histopathological examination.

\section{Results}

Eighty-nine enlarged IMLNs were surgically retrieved from $30.7 \%$ (83/270) of the patients ( 73 in DBR and 10 in IBR) with an age range of 29 to 77 years (median: 45). Eighty six were incidentally encountered and surgically obtained during 307 free-flap reconstruction procedures in 270 patients ( 255 unilateral and 26 bilateral), with a flap success rate of $98.5 \%$. Preoperative staging CT scan for patients mainly undergoing delayed reconstruction was performed in $27.7 \%$ (75/270). This detected isolated radiologically enlarged IMLNs in 4\% (3/75) and confirmed on PET/CT scan (two warm spots and one hot spot). They were subsequently biopsied (two needle-guided CT scan and one mediastinoscope) with no evidence of metastases in any of them. No additional morbidity was associated when CT-guided biopsy was performed; however, when not feasible or deemed risky, biopsy under vision with mediastinoscope was performed ( - Fig. 2). The median size of the IMLN was $1 \mathrm{~cm}$ (range: $0.5-2$ ), with all lymph nodes retrieved from the second and third intercostal spaces, respectively (- Fig. 3). 


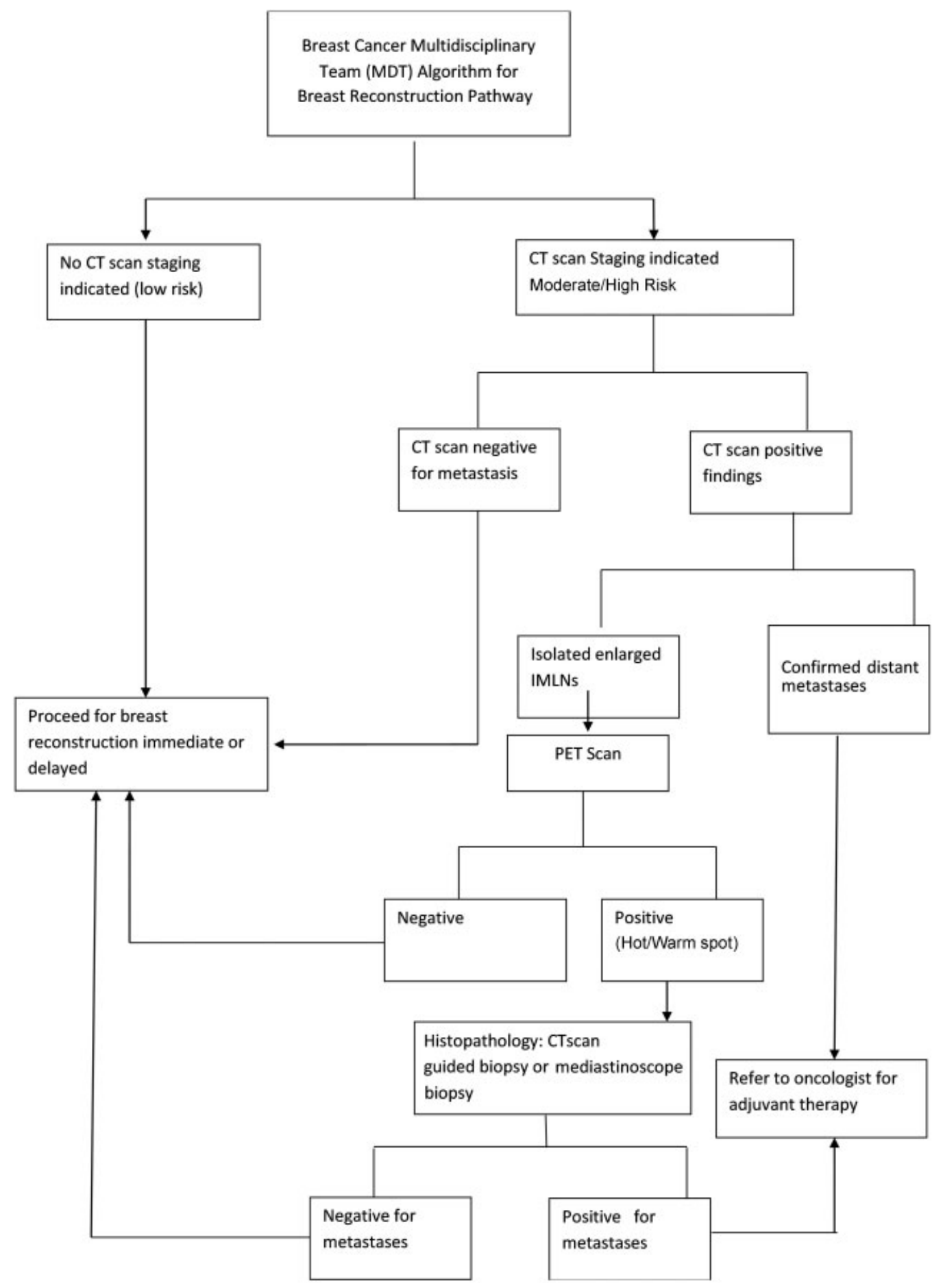

Fig. 1 Diagram demonstrating the Breast Cancer Multidisciplinary Team (MDT) Algorithm for Breast Reconstruction Pathway with special attention to the management plan for incidental radiologically detected internal mammary lymph node enlargement.

IMLN metastases were confirmed in $8.4 \%(7 / 83)$ of the patients in whom IMLNs were retrieved (5 DBR - 2 IBR) and 2.5\% (7/270) of the whole cohort of breast cancer patients undergoing freeflap reconstruction with subsequent modification of the proposed adjuvant therapy. This included second-line chemotherapy and radiotherapy to the supraclavicular area and internal mammary chain, in addition to hormonal treatment. Other histopathological diagnosis included silicone granulomas from previous implant surgery $(n=14)$, sinus histiocytosis
( $n=8)$, and reactive lymph nodes $(n=60)$. One node was harvested in 75 patients, two nodes in 4 , and three nodes in 2 . Macroscopically, there was no significant difference between the metastatic and benign retrieved IMLNs. The free autologous tissue transfer included free muscle-sparing transverse rectus abdominis myocutaneous (MSTRAM) type I/II flap $(n=301)$, superior gluteal artery perforator flap $(n=3)$, and transverse myocutaneous gracilis flap $(n=3)$ in an immediate setting $(n=192)$ or delayed setting $(n=115)$ 

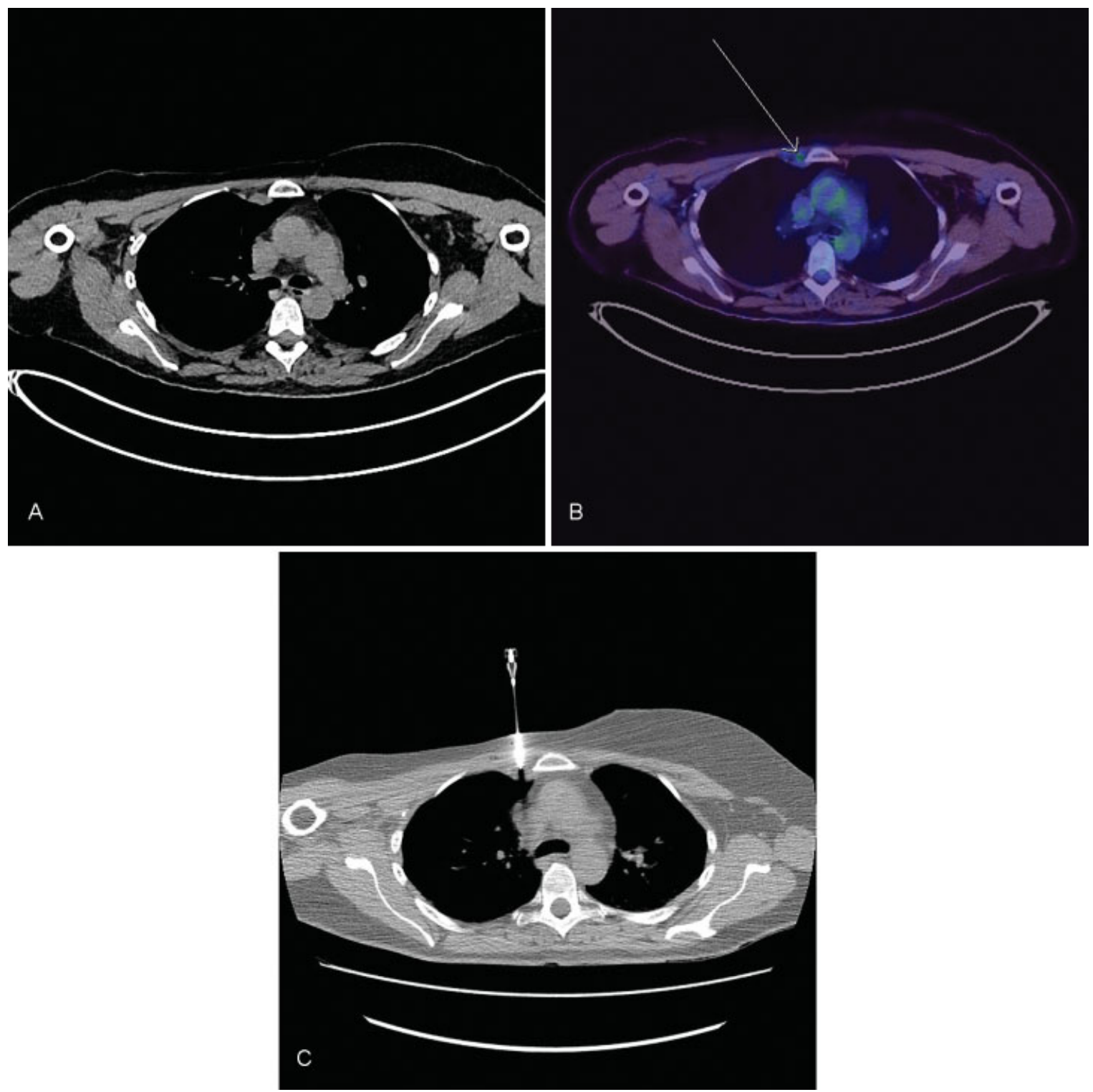

Fig. 2 (A) A preoperative computed tomography (CT) scan staging prior to delayed reconstruction revealing an isolated enlarged isolated internal mammary lymph node (IMLN). (B) A positron emission tomography (PET)/CT scan performed to determine the nature of the enlarged IMLN and also exclude any distant metastases; this scan revealed a hot spot that required further assessment with guide biopsy. (C) CT scan guided biopsy is performed to detect the nature of warm/hot spots on PET/CT scan.

postmastectomy. The IMVP was always the recipient pedicle of choice. The duration between the primary diagnosis and the DBR ranged from 12 to 84 months (median: 30 ).

The tumor, patient characteristics, and the follow-up outcomes for the seven patients (7/270) diagnosed with incidental positive IMLNs are summarized in - Table 1. The original tumor site involved the medial quadrants of the breast in nearly $86 \%$ (6/7) patients with concurrent disease in the axilla also present in $86 \%(6 / 7)$ at their initial presentation. One patient showed signs of IMLN disease progression at 16 months with subsequent chest wall involvement despite adjuvant chemotherapy. Subsequently, she underwent palliative resection and reconstruction using sternal plates and pectoralis major muscle flap to control symptoms (fungation and pain) and improve quality of life. However, one patient died 14 months later due to systemic disease progression within the follow-up period ( - Fig. 4). A second patient presented with stable bony metastases at 32 months with no evidence of disease progression at 55 months followup. The follow-up period ranged from 3 to 84 months (mean: 42) for the involved IMLN patients.

\section{Discussion}

IMLN metastases in breast cancer can occur at multiple levels; however, the most commonly encountered site in is the second or third intercostal space. ${ }^{15}$ The involvement of the IMLN at primary presentation is $3 \% 16,17$ whereas the recurrence rate is 0.1 to $1.5 \%{ }^{14,15,18}$ Clinical and radiological presentations vary from asymptomatic which could be incidentally found on CT scan during routine follow-up as a localized lump within the chain with or without musculoskeletal erosion or symptomatic 

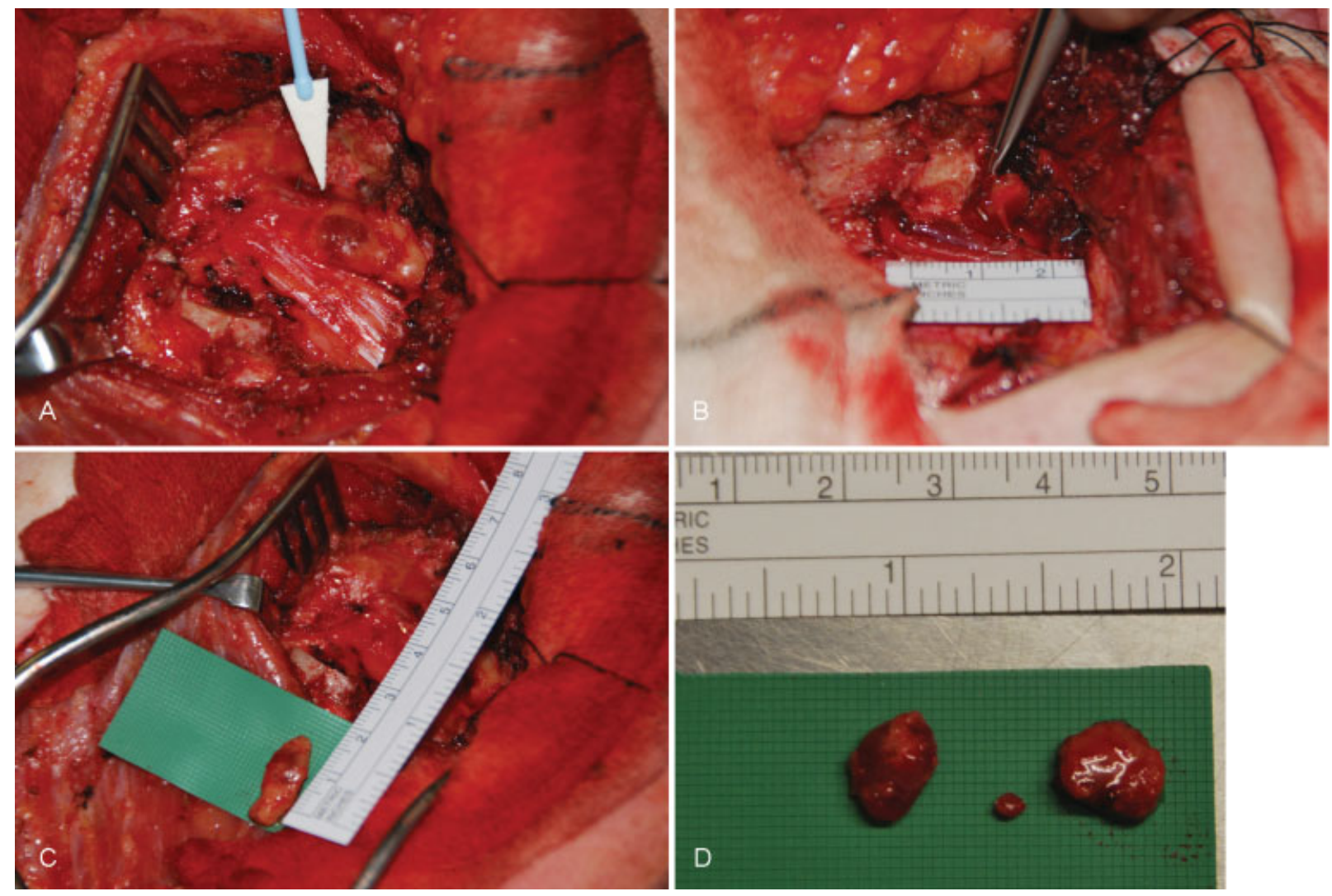

Fig. 3 (A) Intraoperative picture showing an incidental enlarged internal mammary lymph node (IMLN) within the perivascular fat of internal mammary vascular pedicle (recipient vessels) in the third intercostal space during the preparation for microvascular tissue transfer for breast reconstruction. (B) Intraoperative picture demonstrating the dissection of the IMLN with isolation and ligation of its vascular pedicle.

(C) Intraoperative picture postretrieval of a $1 \mathrm{~cm}$ enlarged IMLN. (D) Intraoperative photo revealing multiple retrieved IMLNs with different sizes which could be occasionally encountered.

including sternal or parasternal swelling with concomitant pain or skin involvement including ulceration and fungation. ${ }^{15,19}$ IMLN metastasis is an important prognostic factor in breast cancer patients ${ }^{2,20,21}$; however, concerns over accessibility, morbidity, and survival benefit from IMLN biopsy and dissection have contributed to poor uptake of this technique and lack of consensus regarding the most appropriate or effective forms of investigation and treatment in this group of patients. ${ }^{13,22,23}$ Even with the use of preoperative lymphoscintigraphy, the detection rates were only $25 \%,{ }^{11,16,24}$ with even a lower successful biopsy rate (14\%). Microvascular tissue transfer for breast reconstruction is considered the gold standard, and using the IMVP as the recipient pedicle serves a perfect setting for harvesting the IMLN without any additional morbidity. ${ }^{7,23,25-27}$ The outcome of our series correlates with these previously published studies in which $30 \%$ of the patients had enlarged incidental IMLN harvested of $83 / 270$ (30.7\%) with no additional morbidity. The IMLN metastases are considered forerunner for distant metastases and surrogate of systemic disease, hence the requirement of adjuvant systemic therapy. ${ }^{14}$ Historically, the routine dissection of IMLN has not been universally recommended due to the failure to demonstrate any survival benefit. ${ }^{28,29}$ On the other hand, several studies have demonstrated a significant statistical improvement in locoregional control and overall survival with systemic che- motherapy and radiation to the IMLN chain., ${ }^{7,23,25-27,30}$ In our study, seven patients showed positive IMLN for metastasis, which respectively has had an impact on the overall treatment plan that included either adjuvant chemotherapy or locoregional targeted radiotherapy, highlighting the clinical value of incidentally detected positive IMLN, which has been reported consistently in previous studies. ${ }^{7,23,25-27}$ Within the follow-up period, six of these patients are still alive with no evidence of locoregional or distant metastasis, whereas one patient showed signs of disease progression despite adjuvant chemotherapy. Radiological evidence reported in the literature did show enlarged IMLN predominately detected in the second and third intercostal spaces; however, it can span from the first to fourth intercostal space. ${ }^{15,30}$ From the practical therapeutic point of view, the number of intercostal spaces involved did not have an impact on the locoregional control. ${ }^{30}$ Similarly, the vast majority of the surgically encountered IMLNs were harvested from the second and third intercostal spaces after resecting the third costal cartilage. ${ }^{7,23,25-27}$ Zhang et al reported an overall incidence of $14 \%(112 / 809)$ of involved IMLN in advanced breast cancer by ultrasound (US) at their initial presentation. Biopsy of the IMLN was not routinely performed as most of his patients had simultaneously confirmed metastases in the axillary and supraclavicular region. ${ }^{30}$ Nevertheless, 10/112 (9\%) did undergo fine-needle aspiration cytology (FNAC), 


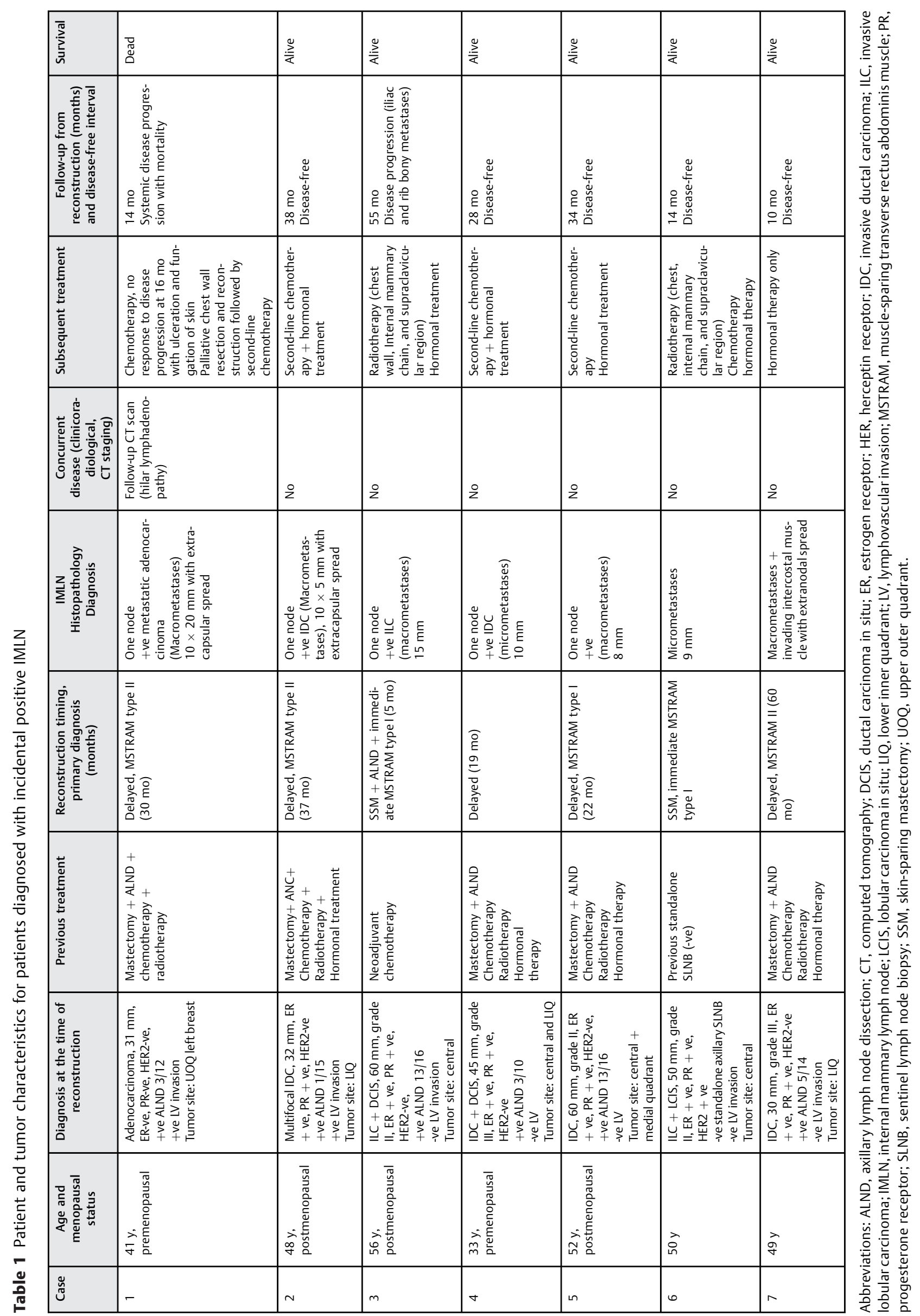



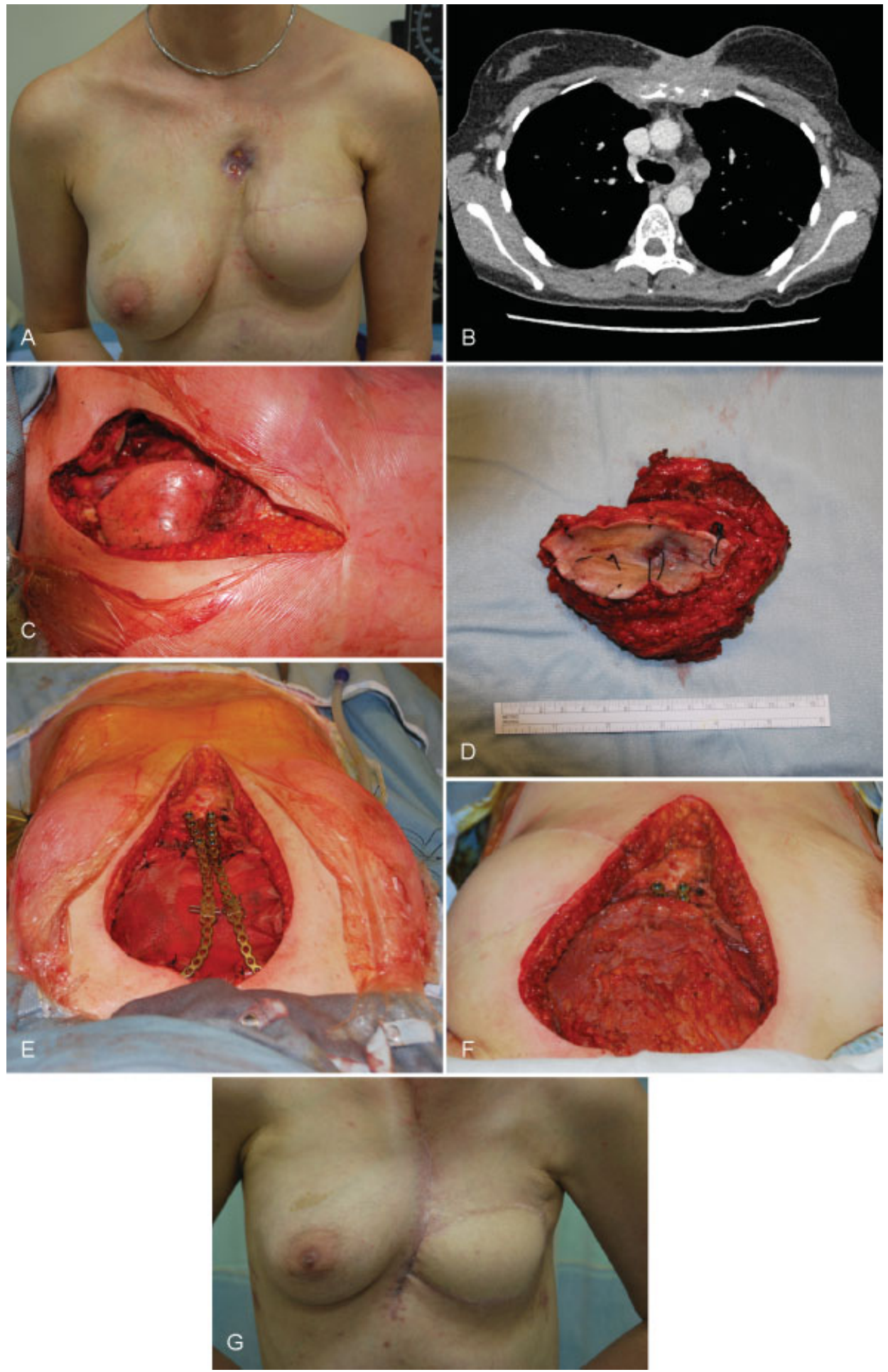

Fig 4 (A) Preoperative picture showing a triple-negative breast cancer patient who underwent mastectomy and delayed reconstruction with free muscle-sparing transverse rectus abdominis myocutaneous (MSTRAM) muscle with an incidental finding of positive malignant internal mammary lymph node (IMLN) during dissection of the internal mammary vascular pedicle. Despite postoperative chemotherapy, disease progression occurred 16 months later with ulceration/skin breakdown discharge and intractable sternal pain. (B) Computed tomography (CT) scan image showing IMLN mass invading the sternum and the adjacent costochondral junctions. (C) Intraoperative picture showing the extent of palliative resection including near total sternectomy and bilateral adjacent costochondral junction with exposure of the intrathoracic contents. (D) Intraoperative picture showing the pathological specimen post en bloc resection. (E) Intraoperative picture showing skeletal chest wall reconstruction achieved with sternal titanium plates + polypropylene mesh. (F) Intraoperative picture showing soft tissue achieved with unilateral left rotational pectoralis major muscle flap followed by primary closure. (G) Post-operative picture ( 5 months) with complete healing of the sternal wound with primary intention and despite the resection the vascularity of the free MSTRAM flap appeared not to be jeopardized. 
representing the rare group of patients who had suspicious IMLN metastases as the only regional nodal involvement. Their study had too few IMLN metastases detected on CT or PET scan, and it was difficult to compare their sensitivity to that of the US, which was proven to be sensitive and cost-effective. Comparatively, worldwide, the majority of the performed breast reconstructions are implant-based $^{31}$; however, they would not benefit from the advantage of surgical accessibility of the IMLN basin. Therefore, radiological investigations depending on the available expertise, including US, CT/PET scan, magnetic resonance imaging (MRI), lymphoscintigraphy, and radiological guided FNAC or biopsy, would be a reasonable approach to assess the IMLN basin without any additional morbidity. That is to provide more accurate staging for therapeutic purposes, especially in medial breast cancers and high-risk patients. ${ }^{29,30}$ In our study, 3/75 (4\%) staging CT scans showed incidental isolated enlarged IMLN, and following our algorithm, subsequent PET/CT scan determined whether a biopsy would be required or not. Cold spots are regarded negative, and those patients would proceed for breast reconstruction as they would be considered nonmetastatic. On the other hand, warm or hot spots underwent CT-guided biopsy and mediastinoscope, which was negative for metastasis in all three patients. If IMLN biopsy had turned out to be positive, then these patients would have been candidates for multimodality adjuvant chemoradiotherapy, and the reconstruction would have deferred to a later stage. Emerging evidence has demonstrated that patients with positive IMLN who were managed with multimodality treatment had more than $50 \%$ disease-free survival and acceptable rates of locoregional control at the IMLN basin. ${ }^{30}$ This correlates with our findings as 2/7 patients (28\%) with positive metastatic IMLN showed signs of disease progression despite multimodality treatment.

Reconstructive surgeons play an important role in treating patients with chest wall involvement facilitating chest wall resection (CWR) and immediate reconstruction in terms of managing pain control, skin involvement, and preventing uncontrolled tumor growth, thereby optimizing long-term outcomes and quality of life in this group of patients. Surgery is considered the cornerstone in isolated locoregional metastases as the effectiveness of chemotherapy and/or radiotherapy as a first line remains unclear. ${ }^{32,33}$ In this study, one patient who previously underwent free MSTRAM for DBR and was proven to have an incidental positive IMLN required palliative CWR and reconstruction with sternal plates and pectoralis major muscle to control locoregional IMLN recurrence 2 years later. Observation from previously published reports demonstrated that proximate collaboration between several disciplines with a multidisciplinary thoracic oncoplastic approach is of paramount importance in devising a comprehensive and safe outcome for this challenging group of patients. ${ }^{34,35}$ Few studies reported a strong relationship between tumor location and the lymphatic drainage, with medial tumors lymphatic drainage been higher to IMLN ${ }^{1,34}$; however, it was not emphasized by other studies. ${ }^{9,32}$ This has led to an ongoing debatable issue that patients with medially located tumors are understaged and receive inadequate oncological treat- ment. ${ }^{36-38}$ It is worth noting that $88 \%$ (6/7) patients presented initially with medial tumors. Endorsement of a proposition plan for diagnosis and management of IMLN metastases remains an undetermined multidisciplinary dilemma, which emphasizes the role of microvascular surgeons to exuviate a better understanding on the natural course and implications on treatment and outcomes. There are limitations to this study. This is a single-institution retrospective study design with a relatively small number of patients with positive IMLN and radiologically detected enlarged IMLN on prereconstruction CT scan staging, possibly as a result of the rarity of the presentation. This may indicate that the cohort is underpowered to achieve any statistical results. Furthermore, more studies are required to objectively highlight the indications for prebreast reconstruction radiological investigations as there is no actual consensus to date for performing preoperative radiological based staging in patients undergoing IBR or DBR.

This study is driven to imperatively emphasize the significance of correlating the clinicopathological and radiological features, focusing on the importance of sampling any incidentally enlarged IMLN encountered during microvascular tissue transfer using the IMVP for breast reconstruction and performing preoperative CT scan staging prior to breast reconstruction, especially in high-risk patients and medial tumors. Eventually, this will enable better assessment of the IMLN basin, especially with the growing evidence of the impact of IMLN metastases in determining a comprehensive oncological treatment plan including chemotherapy, radiotherapy, and CWR with reconstruction.

\section{Note}

Part of this work has been presented as an oral presentation in the Association of Breast Surgery Meeting, 2015, Bournemouth, United Kingdom, with the abstract published in the official journal, European Journal of Surgical Oncology, June 2015, Vol.41(6):55-58.

\section{Conflict of Interest}

None.

\section{References}

1 Koo MY, Lee SK, Bae SY, et al. Long-term outcome of internal mammary lymph node detected by lymphoscintigraphy in early breast cancer. J Breast Cancer 2012;15(01):98-104

2 Veronesi U, Cascinelli N, Greco M, et al. Prognosis of breast cancer patients after mastectomy and dissection of internal mammary nodes. Ann Surg 1985;202(06):702-707

3 Paredes P, Vidal-Sicart S, Zanón G, et al. Clinical relevance of sentinel lymph nodes in the internal mammary chain in breast cancer patients. Eur J Nucl Med Mol Imaging 2005;32(11): 1283-1287

4 Carcoforo P, Sortini D, Feggi L, et al. Clinical and therapeutic importance of sentinel node biopsy of the internal mammary chain in patients with breast cancer: a single-center study with long-term follow-up. Ann Surg Oncol 2006;13(10):1338-1343

5 Mansel RE, Goyal A, Newcombe RG; ALMANAC Trialists Group. Internal mammary node drainage and its role in sentinel lymph node biopsy: the initial ALMANAC experience. Clin Breast Cancer 2004;5(04):279-284 
6 Chagpar AB, Kehdy F, Scoggins CR, et al. Effect of lymphoscintigraphy drainage patterns on sentinel lymph node biopsy in patients with breast cancer. Am J Surg 2005;190(04):557-562

7 Wright EJ, Momeni A, Kraneburg UM, et al. Clinical significance of internal mammary lymph node biopsy during microsurgical breast reconstruction: review of 264 cases. Plast Reconstr Surg 2016;137(06):917e-922e

8 Long H, Lin Z, Situ D, Ma G, Zheng Y, Rong T. Stage migration and therapy modification after thoracoscopic internal mammary lymph node dissection in breast cancer patients. Breast 2011; 20(02):129-133

9 Caudle AS, Yi M, Hoffman KE, et al. Impact of identification of internal mammary sentinel lymph node metastasis in breast cancer patients. Ann Surg Oncol 2014;21(01):60-65

10 Yao MS, Kurland BF, Smith AH, et al. Internal mammary nodal chain drainage is a prognostic indicator in axillary node-positive breast cancer. Ann Surg Oncol 2007;14(10):2985-2993

11 van der Ent FW, Kengen RA, van der Pol HA, Povel JA, Stroeken HJ, Hoofwijk AG. Halsted revisited: internal mammary sentinel lymph node biopsy in breast cancer. Ann Surg 2001;234(01):79-84

12 Madsen E, Gobardhan P, Bongers V, et al. The impact on postsurgical treatment of sentinel lymph node biopsy of internal mammary lymph nodes in patients with breast cancer. Ann Surg Oncol 2007;14(04):1486-1492

13 Chen RC, Lin NU, Golshan M, Harris JR, Bellon JR. Internal mammary nodes in breast cancer: diagnosis and implications for patient management - a systematic review. J Clin Oncol 2008; 26(30):4981-4989

14 Cranenbroek S, van der Sangen MJ, Kuijt GP, Voogd AC. Diagnosis, treatment and prognosis of internal mammary lymph node recurrence in breast cancer patients. Breast Cancer Res Treat 2005;89(03):271-275

15 Chen L, Gu Y, Leaw S, et al. Internal mammary lymph node recurrence: rare but characteristic metastasis site in breast cancer. BMC Cancer 2010;10:479

16 Farrús B, Vidal-Sicart S, Velasco M, et al. Incidence of internal mammary node metastases after a sentinel lymph node technique in breast cancer and its implication in the radiotherapy plan. Int J Radiat Oncol Biol Phys 2004;60(03):715-721

17 Bourre JC, Payan R, Collomb D, et al. Can the sentinel lymph node technique affect decisions to offer internal mammary chain irradiation? Eur J Nucl Med Mol Imaging 2009;36(05):758-764

18 Fowble B, Solin LJ, Schultz DJ, Goodman RL. Frequency, sites of relapse and outcome of regional node failures following conservative surgery and radiation for early breast cancer. Int J Radiat Oncol Biol Phys 1989;17(04):703-710

19 Scott WW Jr, Fishman EK. Detection of internal mammary lymph node enlargement: comparison of CT scans and conventional roentgenograms. Clin Imaging 1991;15(04):268-272

20 Klauber-DeMore N, Bevilacqua JL, Van Zee KJ, Borgen P, Cody HS III. Comprehensive review of the management of internal mammary lymph node metastases in breast cancer. J Am Coll Surg 2001;193(05):547-555

21 Sugg SL, Ferguson DJ, Posner MC, Heimann R. Should internal mammary nodes be sampled in the sentinel lymph node era? Ann Surg Oncol 2000;7(03):188-192

22 Purushotham AD, Cariati M. Internal mammary nodes and breast cancer. Br J Surg 2005;92(02):131-132
23 Yu JT, Provenzano E, Forouhi P, Malata CM. An evaluation of incidental metastases to internal mammary lymph nodes detected during microvascular abdominal free flap breast reconstruction. J Plast Reconstr Aesthet Surg 2011;64(06):716-721

24 Johnson N, Soot L, Nelson J, et al. Sentinel node biopsy and internal mammary lymphatic mapping in breast cancer. Am J Surg 2000; 179(05):386-388

25 Andree C, Schmidt VJ, Munder BI, et al. Detecting of breast cancer metastasis by means of regional lymph node sampling during autologous breast reconstruction-a screening of 519 consecutive patients. Med Sci Monit 2012;18(10):CR605-CR610

26 Schaverien MV, Purdie CA, Munnoch DA. Clinical value of internal mammary lymph node metastases found incidentally during free flap recipient vessel exposure. Eur J Surg Oncol 2013;39(06): 608-612

27 Wong KY, Yu JT, Mahler-Araujo B, Forouhi P, Malata CM. Opportunistic internal mammary lymph node biopsy during microvascular free flap breast reconstruction: a useful technique? Int J Surg 2013;11(09):1027-1028

28 Veronesi U, Marubini E, Mariani L, Valagussa P, Zucali R. The dissection of internal mammary nodes does not improve the survival of breast cancer patients. 30 -year results of a randomised trial. Eur J Cancer 1999;35(09):1320-1325

29 Veronesi U, Arnone P, Veronesi P, et al. The value of radiotherapy on metastatic internal mammary nodes in breast cancer. Results on a large series. Ann Oncol 2008;19(09):1553-1560

30 Zhang YJ, Oh JL, Whitman GJ, et al. Clinically apparent internal mammary nodal metastasis in patients with advanced breast cancer: incidence and local control. Int J Radiat Oncol Biol Phys 2010;77(04):1113-1119

31 Ricci JA, Epstein S, Momoh AO, Lin SJ, Singhal D, Lee BT. A metaanalysis of implant-based breast reconstruction and timing of adjuvant radiation therapy. J Surg Res 2017;218:108-116

32 van Geel AN, Wouters MW, van der Pol C, Schmitz PI, Lans T. Chest wall resection for internal mammary lymph node metastases of breast cancer. Breast 2009;18(02):94-99

33 Petrella F, Radice D, Borri A, et al. Chest wall resection and reconstruction for locally recurrent breast cancer: from technical aspects to biological assessment. Surgeon 2016;14(01):26-32

34 Khalil HH, Malahias MN, Balasubramanian B, et al. Multidisciplinary oncoplastic approach reduces infection in chest wall resection and reconstruction for malignant chest wall tumors. Plast Reconstr Surg Glob Open 2016;4(07):e809

35 Basta MN, Fischer JP, Lotano VE, Kovach SJ. The thoracoplastic approach to chest wall reconstruction: preliminary results of a multidisciplinary approach to minimize morbidity. Plast Reconstr Surg 2014;134(06):959e-967e

36 Huang O, Wang L, Shen K, et al. Breast cancer subpopulation with high risk of internal mammary lymph nodes metastasis: analysis of 2,269 Chinese breast cancer patients treated with extended radical mastectomy. Breast Cancer Res Treat 2008;107(03): 379-387

37 Colleoni M, Zahrieh D, Gelber RD, et al. Site of primary tumor has a prognostic role in operable breast cancer: the international breast cancer study group experience.J Clin Oncol 2005;23(07):1390-1400

38 Noushi F, Spillane AJ, Uren RF, Gebski V. Internal mammary lymph node metastasis in breast cancer: predictive models to assist with prognostic influence. Breast 2011;20(03):278-283 